\title{
La Interculturalidad como Competencia en Educación Superior: Validación de un Instrumento con Alumnado Universitario
}

\author{
Interculturality as a Competence in Higher Education: Validation of an \\ Instrument with University Students \\ A interculturalidade como competência no Ensino Superior: validação de um \\ instrumento com estudantes universitários
}

Francisco Javier de Santos Velasco* 0000-0001-7107-5323

Departamento de Educación Continua, Dirección de Gestión del Talento, Vicerrectoría de Educación Continua, Instituto Tecnológico y de Estudios Superiores de Monterrey, México

$\nabla$

Recibido: 05-04-18 Revisado:08-06-18 Aceptado:05-10-18 Publicado: 20-12-18

- Resumen.Este artículo, basado en los resultados de un estudio de tesis doctoral aborda dos aspectos: la adaptación y validación de un instrumento para medir la sensibilidad intercultural del alumnado universitario y la relación de la medición de la escala de sensibilidad intercultural frente al perfil de experiencia del alumnado universitario ante lo internacional y/o lo multicultural. Se aplicó el instrumento a 280 alumnos de la Universidad Pompeu Fabra en Barcelona, España. Los resultados del análisis de las características técnicas de la escala indican que cuenta con validez de contenido y que las puntuaciones del instrumento poseen confiabilidad. Se encontró que todas las variables que tienen relación con la experiencia directa ante lo internacional y/o multicultural aparecen con diferencia significativa, excepto la variable número de profesores extranjeros, lo cual apoya investigaciones que han encontrado que el aprendizaje de la competencia intercultural es un proceso activo de aprendizaje permanente a lo largo de toda la vida, con un alto componente
Palabras Clave:

Enseñanza

Superior,

Educación

Universal,

Educación

Intercultural,

Comunicación

Intercultural 
vivencial, es decir, que se lleva a cabo sobre todo a través de la vivencia o experiencia directa con "el otro diferente", sea en interacciones de uno a uno, o bien, grupales; sea en el propio país o en el extranjero.

- Abstract.This article, based on the results of a doctoral thesis study, deals with two aspects: the adaptation and validation of an instrument to measure the intercultural sensitivity of university students and the relationship between the measurement of the intercultural sensitivity scale versus the experience profile of university students in the face of international and/or the multicultural experiences. The instrument was applied to 280 students from Universidad Pompeu Fabra in Barcelona, Spain. The results of the analysis of the technical characteristics of the scale indicate that it has content validity and that the instrument scores are reliable. It was found that all variables related to direct international and/or multicultural experience appear with a significant difference, except for the variable referring to number of foreign teachers, which supports research works that have found that the learning of the intercultural competence is an active process of constant learning throughout life, with a high experiential component, i.e., that it is carried out mainly through direct experience with "the other different," either in one-to-one or group interactions, either in their own country or abroad.

- Resumo. Este artigo, baseado nos resultados de um estudo de tese doutoral, aborda dois aspectos: a adaptação e validação de um instrumento para medir a sensibilidade intercultural de estudantes universitários e a relação da medição da escala de sensibilidade intercultural versus o perfil de experiência do alunado universitário diante do internacional e/ou o multicultural. Aplicou-se o instrumento a 280 alunos da Universidade Pompeu Fabra em Barcelona, Espanha. Os resultados da análise das características técnicas da escala indicam que conta com validez de conteúdo e que as pontuações do instrumento possuem confiabilidade. Encontrou-se que todas as variáveis que têm relação com a experiência direta diante do internacional e/ou multicultural aparecem com diferença significativa, a exceção da variável número de professores estrangeiros, que sustenta pesquisas cujos resultados indicam que a aprendizagem da competência intercultural é um processo ativo e permanente com um alto componente vivencial, isto é, que se realiza através da vivência ou experiência direta com "o outro diferente", ora em interações um a um, ou bem grupais; ora no próprio pais ou no exterior.
Keywords:

higher education, universal education, intercultural education, intercultural communication

Palavras-chave: ensino superior, educação universal, educação intercultural, comunicação intercultural 


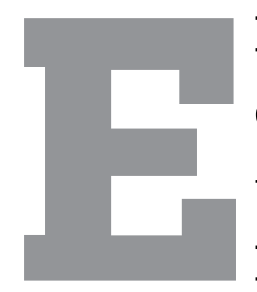

lestudio de la interculturalidad como competencia en la educación superior ha tenido diferentes enfoques. En el contexto de la mayoría de los países de América Latina, tanto desde las universidades públicas como privadas, se han enfocado en abordar la necesidad de incluir en los estudios universitarios a grupos sociales minoritarios como grupos indígenas, migrantes y afrodescendientes.

Por tanto, dicho enfoque ha tenido un objetivo de equidad educativa, a fin de evitar la discriminación histórica de que dichos grupos han sido objeto y favorecer el acceso de tales poblaciones al nivel de la educación superior, dando origen en algunos países al diseño de políticas públicas, de programas de becas; a la creación de instituciones de Educación Superior Intercultural (Universidades Interculturales) o al diseño de programas de formación de profesores o educadores, con el fin de cumplir con tal propósito. (Ladrón de Guevara, 2014; Pascual-Ortega, D. A. (2013); Mendoza, Blanca (2013); Bertely Busquets, M. (2011); Mato, D. (2009); Mato, D. (2009a); Mato, D. (2008).

En años más recientes, se ha ampliado el enfoque de los grupos minoritarios a todo el alumnado universitario, a través de la transversalización de la interculturalidad en la formación integral del mismo, así como a los ambientes virtuales de aprendizaje (Cano Barrios, J. et al (2016); Ladrón de Guevara, (2014).

En el contexto europeo, los estudios se han orientado hacia el fortalecimiento del espacio europeo de educación superior, tanto desde una perspectiva de ciudadanía, como desde el enfoque de movilidad laboral, a fin de que el alumnado universitario se sienta parte de la Unión Europea, y que se fortalezca la competencia intercultural, que le permita moverse laboralmente y ser competente en cualquier país que trabaje. (Martínez-Lirola, M. (2018); Pozo-Vicente, C. et al (2012); Edwards Schachter, M. y López Santiago, M. (2008) y Bartolomé, M. (2002). El presente estudio realizado con alumnado universitario en España, se realiza dentro del marco del enfoque europeo (De Santos, F.J. (2004).

La competencia intercultural es una de las competencias transversales necesarias para que los profesionales y empleados del futuro puedan vivir y desempeñarse mejor en las organizaciones de hoy y del mañana. Ya no es necesario salir al extranjero para encontrarse con lo multicultural, ya que este está en casi todas partes, por lo que la capacidad de actuar internacionalmente e interculturalmente y de poseer cualificaciones internacionales e interculturales, se convierten en competencias clave para buena parte de la mano de obra, aunque esta no se desplace en realidad más allá de sus fronteras.

Ante este contexto laboral cada vez más globalizado e interconectado, las instituciones de educación superior se ven en la necesidad de responder con una oferta educativa que responda a la diversidad de necesidades de las sociedades multiculturales actuales y futuras, donde la formación del profesorado y la del alumnado universitario son prioritarias, con el objetivo de preparar a los estudiantes universitarios para el mundo laboral, cada vez más relacionado con 
lo internacional y multicultural. (Espinosa Romero, M. (2009); Villar, S. A. (2008); Medina R. A.; Rodríguez M. A. e Ibáñez, A. coords. (2005).

El estudio del tema planteado vendría a complementar el conocimiento empírico en el ámbito de la educación universitaria, del mundo académico hispano parlante, tanto en el contexto europeo como hispanoamericano.

\section{La competencia intercultural}

De acuerdo con Guo-Ming Chen, profesor del Departamento de Estudios de la Comunicación de la Universidad de Rhode Island, en los Estados Unidos, quien aborda el concepto de competencia comunicativa intercultural como un concepto paraguas, el cual comprende las habilidades cognitiva, afectiva y conductual de los interactuantes en el proceso de comunicación intercultural.

Al aspecto cognitivo de la competencia comunicativa intercultural le llama conciencia intercultural. Al aspecto afectivo de la competencia le denomina sensibilidad intercultural y al aspecto conductual lo nombra efectividad intercultural.

El presente estudio se centrará en el aspecto de la sensibilidad intercultural, el cual se refiere a el deseo que motiva a las personas a conocer, comprender, apreciar y aceptar las diferencias entre culturas. Para medir dicho aspecto, Chen \& Starosta (2000) diseñaron y validaron una escala, la "Intercultural Sensitive Scale" (ISS), la cual es motivo de adaptación y aplicación en el presente estudio.

\section{Objetivos}

a) Traducir al idioma español y adaptar a la cultura española la escala denominada "Intercultural Sensitivity Scale" (ISS)

b) Diseñar un cuestionario, donde se incluye la escala, así como las variables de contexto del alumnado (perfil de experiencia del alumnado en ámbitos internacionales y/o multiculturales).

c) Medir el nivel de sensibilidad intercultural (actitud del alumnado universitario hacia el hecho de interactuar con personas de diferente cultura), mediante la escala adaptada, y analizar si existe diferencia de medias significativa entre la variable objeto de estudio (sensibilidad intercultural) vs. las variables de contexto del alumnado (perfil de experiencia del alumnado en ámbitos internacionales y/o multiculturales).

d) Realizar estudios de fiabilidad y validez de contenido de la escala adaptada. 


\section{MÉTODO}

\section{Participantes}

Alumnado de la Universidad Pompeu Fabra de Barcelona. Participaron 280 integrantes del alumnado regular de cuarto año de licenciatura, integrantes de varias titulaciones de la Universidad, siendo el 89\% del total de la muestra estudiada los alumnos de las Licenciaturas en Administración y Dirección de Empresas (ADE), así como la Licenciatura en Ciencias Políticas y Administración (CPA).

\section{Instrumento}

Se utilizó el instrumento denominado Intercultural Sensitivity Scale (ISS), desarrollado por Chen \& Starosta (2000). La escala original tipo Likert de cinco niveles consta de 24 ítems, los cuales están distribuidos en cinco dimensiones o factores, a saber: a) Involucramiento en la interacción; b) respeto por las diferencias culturales, c) confianza durante la interacción, d) disfrute de la interacción y e) atención durante la interacción.

\section{Procedimiento}

- Traducción y adaptación de la escala

a) Traducción de la escala del inglés al español (Escala de Sensibilidad Intercultural)

b) Adaptación cultural de la redacción de cada uno de los ítems, elaborando de 2 a 3 opciones de ítems por cada ítem original. Selección de las opciones de ítems que se consideró que correspondían a una mejor traducción y/o adaptación cultural.

- Diseño del cuestionario

c) Una vez que se tuvo la escala traducida y adaptada, se seleccionaron 20 variables de contexto, de las cuales 17 están relacionadas con la familiaridad, en el plano experiencial, con el tema de la multiculturalidad. Con dichas variables se completó el diseño del instrumento y frente a las mismas se realizó el análisis estadístico para verificar la existencia o no de diferencias significativas.

Las variables seleccionadas de exposición a lo multicultural fueron:

Nacionalidad propia, la del padre y la de la madre

Estudiar o haber estudiado otras lenguas diferentes a la materna/paterna

Experiencias internacionales

- $\quad$ Haber vivido en el extranjero

- Haber viajado al extranjero

- Haber participado en programas de intercambio estudiantil al extranjero

Experiencias con "lo extranjero", "internacional" o "multicultural" en su propio país:

- Vivir o haber vivido con alguien de origen extranjero

- Tener o haber tenido amigos extranjeros 
- $\quad$ Número de profesores extranjeros en la Universidad

- Participación voluntaria en eventos diversos relacionados con otros países o culturas (seminarios, fiestas religiosas, conciertos, ferias o bazares, exposiciones, etc.)

d) Se realizó un proceso piloto con seis alumnos(as) del 3ro y 4to años de la UPF, con el fin de validar el instrumento, tanto en la adaptación de la escala (traducción inglés-español y adaptación cultural), como para verificar comprensión de lenguaje de los ítems de la escala (palabras y/o expresiones empleadas), así como claridad de las instrucciones.

e) Partiendo de los resultados del piloto, se realizaron los ajustes sugeridos al instrumento (se corrigió la redacción de cuatro ítems de la escala y se realizaron correcciones de redacción en tres preguntas de exposición a lo multicultural).

- $\quad$ Aplicación del instrumento

Se aplicó, de manera individual, a 280 integrantes del alumnado universitario. El detalle de las poblaciones y muestras se detallan en la Tabla 1.

Tabla 1.

Muestras alumnado

\begin{tabular}{|c|l|}
\hline POBLACION & \multicolumn{1}{|c|}{ MUESTRA } \\
\hline Alumnado ADE 4to año & \multicolumn{1}{|c|}{ Grupo 1 ADE (208 alumnos) } \\
(252 matriculados y 160 habitual) & Licenciatura ADE \\
Materia optativa RH I & $-\quad$ 4to curso materia troncal (143) \\
(185 matriculados y 90 habitual) & $-\quad$ 3ro y 4to curso materia optativa RH (65) \\
Alumnado Ciencias Políticas y Administración & $\begin{array}{l}\text { Grupo 2 CPA (41 alumnos) } \\
\text { (CPA) 4to año } \\
\text { (60 matriculados y 45 habitual) }\end{array}$ \\
& $\begin{array}{l}\text { (Grupo contraste vs. ADE) } \\
\text { Grupo 3 No ADE (31 alumnos) } \\
\text { no Licenciatura ADE }\end{array}$ \\
&
\end{tabular}

A través de una guía de codificación con 65 variables, se elaboró base de datos con las 280 encuestas y se procedió a realizar los estudios de estadística descriptiva, así como de estadística inferencial, de acuerdo con los objetivos propuestos, con el apoyo del paquete estadístico SPSS.

Asimismo, se realizaron análisis de las características técnicas de la escala (Fiabilidad y Validez de contenido de la escala). 


\section{RESULTADOS}

\section{Análisis de las características técnicas de la escala}

Fiabilidad de la escala

A fin de verificar la consistencia interna del instrumento, se realizó el análisis de fiabilidad, mediante el coeficente alpha de Cronbach, obteniéndose una alpha de.83, ligeramente inferior al alpha obtenida en la escala original que fue del .86, por lo que puede decirse que el instrumento traducido y adaptado tiene fiabilidad alta.

Validez de contenido de la escala

Se realizaron estudios estadísticos para analizar la validez de contenido del instrumento, a través de las siguientes pruebas: a) índice de homogeneidad de los items de toda la escala e b) índice de discriminación de los ítems.

Índice de homogeneidad de los ítems de la escala

De los 24 ítems de la escala, prácticamente todos obtienen una correlación significativa con el total de la escala, y por tanto parecen adecuados, a excepción del ítem 15, el cual obtuvo signo negativo, y podría ser objeto de modificación o rechazo. Puede concluirse que la escala como un todo posee una validez de contenido significativa, ya que de los 24 items, solo 1 (4.1\%) obtuvo un índice de homogeneidad negativo.

Índice de discriminación de los ítems de la escala

Se obtuvieron losíndices de discriminación a través de la prueba t de student (comparando las medias de grupos extremos al 50\%, es decir dividiendo al alumnado por su grado de actitud en dos mitades), así como a través de las correlaciones, encontrándose, que el único ítem menos discriminativo en ambas pruebas es el ítem 15, el cual al obtener una discriminación negativa quiere decir que el grupo inferior tiene una media más alta que el grupo superior, por lo que habría que pensar quizá en modificarlo o suprimirlo. Puede concluirse que la escala es mayormente discriminativa, excepto en 1 ítem (4.1\%) que obtuvo un resultado no discriminativo.

\section{Medición de la sensibilidad intercultural del alumnado universitario}

La distribución de las puntuaciones de la escala obtenidas por el alumnado de los tres grupos se ajusta a una distribución normal, lo cual indica que la escala mide adecuadamente la actitud que pretende (actitud del alumnado universitario hacia el hecho de interactuar con personas de diferente cultura). Si partimos de que la puntuación mínima posible de la escala es de 24 puntos (que mostraría una actitud en contra) y la máxima de 120 puntos (que mostraría una actitud muy favorable), siendo la puntuación de 72 la que mostraría una actitud indiferente o indecisa.

En el Grupo ADE se obtuvo una distribución de la actitud que va de los 56 a los 116 puntos, siendo la media de 95.19 puntos y la desviación típica de 9.08, es decir, entre 2.33 y 4.83 con una media de 3.96, lo cual podría esquematizarse de esta manera: 


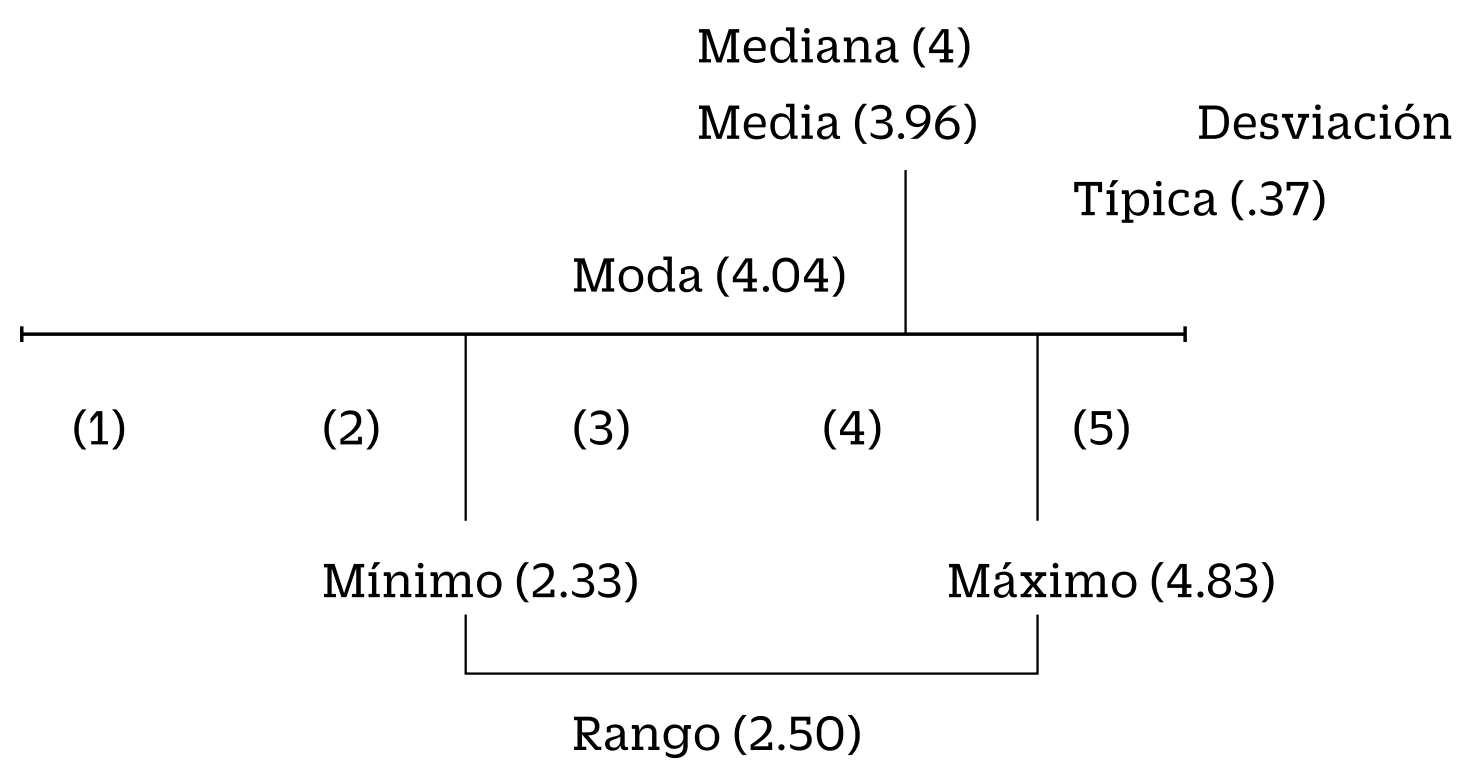

En el Grupo CPA se obtuvo una distribución de la actitud que va de los 83 a los 112 puntos, siendo la media de 97.07 puntos y la desviación típica de 7.9, es decir, entre 3.45 y 4.66 con una media de 4.04, lo cual podría esquematizarse de esta manera:

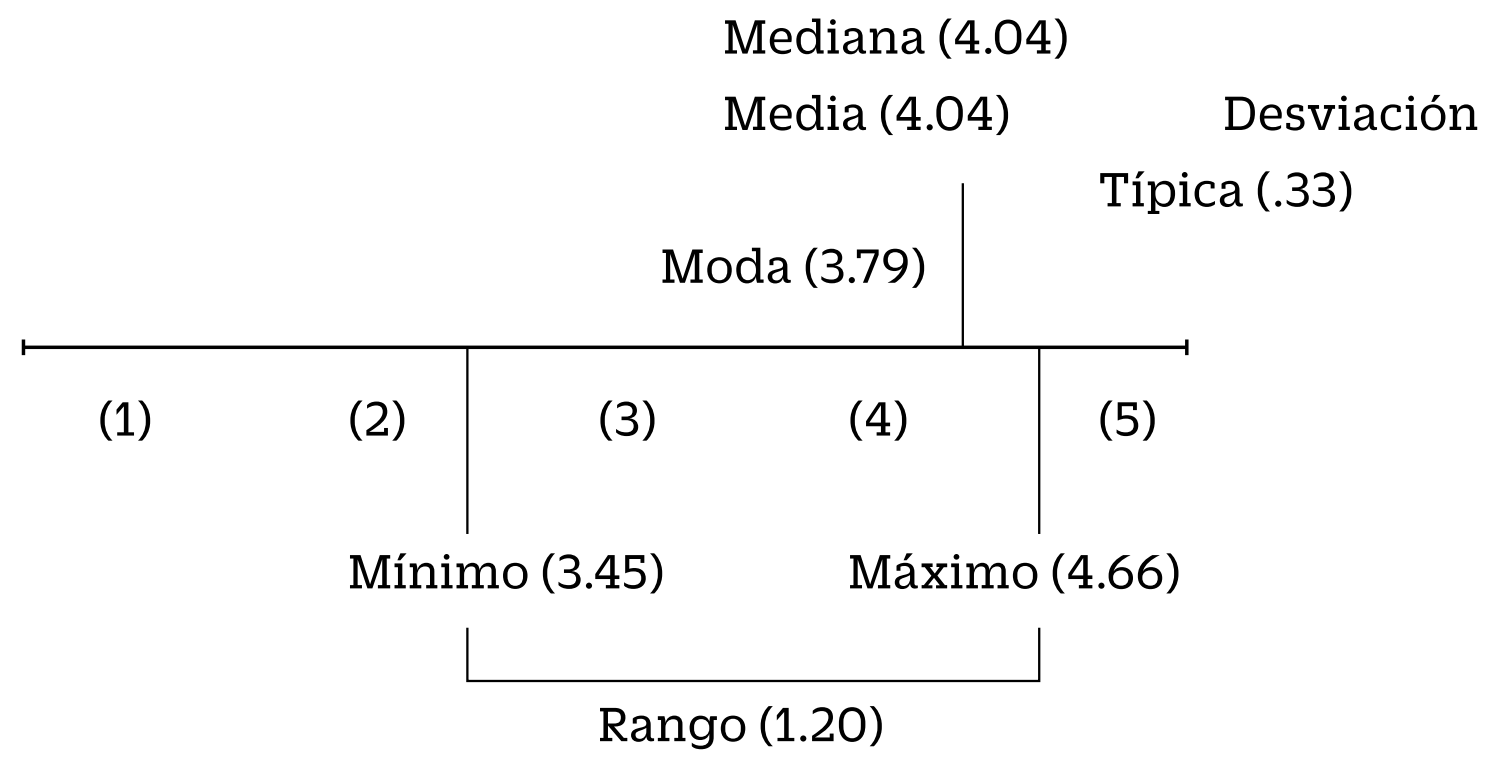

En el Grupo NO ADE se obtuvo una distribución de la actitud que va de los 78 a los 114 puntos, siendo la media de 93.52 puntos y la desviación típica de 9.37, es decir, entre 3.25 y 4.75 con una media de 3.89, lo cual podría esquematizarse de esta manera:

Mediana (3.87)

(1)

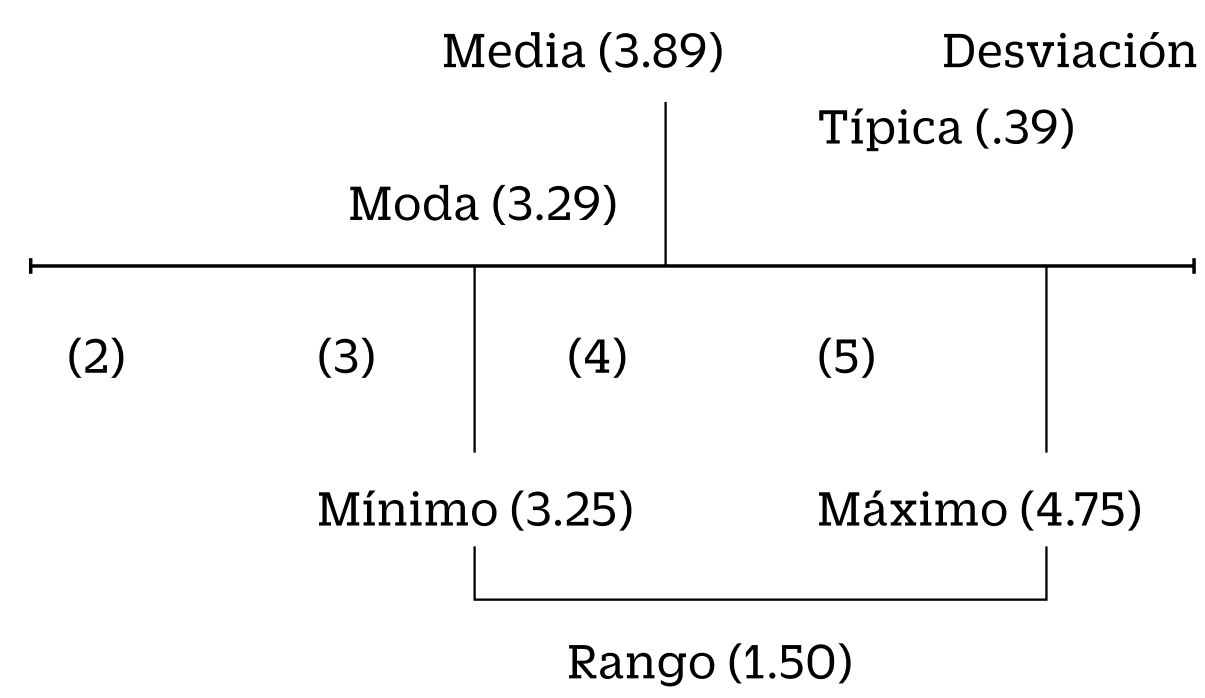


Se podría hacer la siguiente interpretación descriptiva: la puntuación de los tres grupos se distribuye como una curva normal que se inicia antes de la media teórica (72 puntos) en el caso del Grupo ADE (56 puntos) y arriba de la media teórica en los grupos CPA y NO ADE (78 y 83 puntos), por lo que en los tres casos se cubre el espectro de la actitud que va del indiferente o inseguro al muy favorable, situándose en el caso del Grupo ADE desde el no favorable hasta el muy favorable.

No obstante la diferencia en la forma de la dispersión de las puntuaciones entre los grupos, la media de los tres grupos se ubica entre los 93.52 y los 97.07 (3.89 y 4.04), lo cual significa que el alumnado universitario encuestado en los tres grupos tiene una actitud favorable en promedio hacia el hecho de interactuar con personas de diferente cultura, ya que el resultado se ubica en el nivel 4 "de acuerdo", con una mediana cercana también al "de acuerdo", lo cual indica una actitud favorable en promedio.

\section{¿Existe diferencia en la sensibilidad intercultural entre los grupos estudiados?}

A fin de conocer si las diferencias encontradas en las medias de los tres grupos son o no significativas, se realizaron pruebas estadísticas, obteniéndose los siguientes resultados:

a) De acuerdo con la prueba de homogeneidad de varianzas de Levene, no se encontró diferencia significativa en las varianzas de los tres grupos.

b) De acuerdo con la ANOVA, no se encontró diferencia significativa entre las medias de los tres grupos.

No se encontró diferencia significativa entre las medias entre los tres grupos estudiados, lo cual nos indica que el grado de sensibilidad intercultural es similar en los tres grupos. Ello puede deberse a varios motivos, entre otros: a) a un contexto social y universitario similar, en el cual se incluyen los planes de estudios, que en general tienen poca orientación o enfoque internacional y/o intercultural, así como b) a la similitud en la experiencia vital del alumnado integrante de la muestra.

\section{Perfil de experiencia del alumnado universitario ante lo internacional y/o multicultural}

Con relación a las variables de contexto relacionadas con el grado de exposición del alumnado universitario antelo internacional o extranjero, destacaríalossiguientes resultados descriptivos.

a) Más de la mitad (58\%) en todos los grupos NO han vivido en el extranjero, mientras que un tercio (26-35\%) han vivido por un periodo de 1-6 meses.

b) Entre un $32-41 \%$ han viajado más de seis veces al extranjero en los tres grupos y entre un $29-37 \%$ han viajado de 1-3 veces.

c) La mayor parte del alumnado (entre un 65-71\%) de los tres grupos NO ha participado 
en un programa de intercambio al extranjero.

d) Poco más de la mitad (52-56\%) del alumnado de los tres grupos NO han convivido con personas extranjeras en la misma casa o piso.

e) La mayoría (55-77\%) del alumnado NO tienen amigos extranjeros, siendo el mayor porcentaje para el grupo de NO ADE.

\section{Contraste entre la sensibilidad intercultural frente a las variables de contexto de los tres grupos integrados como una sola muestra}

Al no encontrar diferencias significativas entre las medias de los tres grupos, a través de la prueba de homogeneidad de varianzas de Levene, así como con la ANOVA, se decidió realizar el estudio de comparación de medias para cada una de las variables consideradas en el instrumento con los tres grupos de alumnos reunidos como una sola muestra $(n=280)$.

Se realizaron análisis comparativos de medias entre la variable total de la escala (suma de los items) vs. cada una de ellas, utilizando la Prueba $T(t)$ con dos grupos paramétricos; la Prueba de Mann-Whitney ( $m$ - $w$ ) para dos grupos no paramétricos y la Prueba de Kruskal-Wallis $(k-w)$ para más de dos grupos no paramétricos, obteniéndose diferencias significativas en 27 de las 44 comparaciones de medias realizadas, como se muestra en la tabla 2.

\section{Tabla 2}

Comparación de medias con diferencia significativa

\begin{tabular}{|c|c|c|c|}
\hline VARIABLE & GRUPOS COMPARADOS & PRUEBA ESTADÍSTICA & P=SIGNIFICACIÓN (AL 0,05\%) \\
\hline Estudio idioma inglés & Poco vs. Mucho & $\mathrm{t}=-3,494$ & .001 \\
\hline Estudio idioma francés & Nada vs. Mucho & $\mathrm{t}=-2,650$ & .009 \\
\hline Estudio idioma alemán & Todos los grupos & $\mathrm{k}-\mathrm{w}=12,340$ & .002 \\
\hline Estudio idioma italiano & Todos los grupos & $\mathrm{k}-\mathrm{w}=13,821$ & .001 \\
\hline & Idiomas Estado & $\mathrm{t}=-2.535$ & .012 \\
\hline $\begin{array}{c}\text { Idioma materno } \\
\text { Dominio de idiomas }\end{array}$ & $\begin{array}{c}\text { Español vs idiomas } \\
\text { extranjeros }\end{array}$ & $\mathrm{m}-\mathrm{w}=1233,500$ & .000 \\
\hline $\begin{array}{c}\text { Tiempo de vivir en el }<5 \\
\text { extranjero }\end{array}$ & $\begin{array}{c}\text { Nunca vs. 1-6 meses } \\
\text { Todos los grupos }\end{array}$ & $\mathrm{t}=4,319$ & .000 \\
\hline & SI vs. NO & $\mathrm{k}-\mathrm{w}=23,2834$ & .000 \\
\hline Intercambio al extranjero & $1-3$ veces vs. + de 6 veces & $\mathrm{t}=-4,140$ & .000 \\
\hline $\begin{array}{c}\text { No. De viajes al } \\
\text { extranjero }\end{array}$ & Todos los grupos & $\mathrm{k}-\mathrm{w}=26,643$ & .001 \\
\hline
\end{tabular}




\begin{tabular}{|c|c|c|c|}
\hline VARIABLE & GRUPOS COMPARADOS & PRUEBA ESTADÍSTICA & $\mathrm{P}=$ SIGNIFICACIÓN (AL 0,05\%) \\
\hline Vivir con extranjeros & SI vs. NO & $t=-3,235$ & .001 \\
\hline Amigos extranjeros & SI vs. NO & $t=-7,718$ & .000 \\
\hline \multirow{2}{*}{$\begin{array}{c}\text { Seminarios, conferencias } \\
\text { o foros }\end{array}$} & NO vs. Alguna vez & $t=-3,033$ & .003 \\
\hline & Todos los grupos & $\mathrm{k}-\mathrm{w}=10,704$ & .005 \\
\hline \multirow{2}{*}{$\begin{array}{c}\text { Fiestas religiosas o } \\
\text { populares }\end{array}$} & NO vs. Alguna vez & $t=-4,630$ & .000 \\
\hline & Todos los grupos & $\mathrm{k}-\mathrm{w}=18,460$ & .000 \\
\hline \multirow[t]{2}{*}{ Manifestaciones públicas } & NO vs. Alguna vez & $t=-3,484$ & .001 \\
\hline & Todos los grupos & $\mathrm{k}-\mathrm{w}=24,335$ & .000 \\
\hline Conciertos & $\begin{array}{l}\text { NO vs. con cierta } \\
\text { frecuencia }\end{array}$ & $t=-3,156$ & .002 \\
\hline \multirow[t]{2}{*}{ Ferias o Bazares } & NO vs. Alguna vez & $t=-3,149$ & .002 \\
\hline & Todos los grupos & $\mathrm{k}-\mathrm{w}=11,186$ & .004 \\
\hline Exposiciones & $\begin{array}{c}\text { NO vs. con cierta } \\
\text { frecuencia }\end{array}$ & $t=-2,818$ & .006 \\
\hline Participación en eventos & $=>3$ vs. $<3$ & $t=5,143$ & .000 \\
\hline Interés por resultados & SI vs. NO & $t=-2,556$ & .011 \\
\hline $\begin{array}{c}\text { Interés por taller } \\
\text { (sólo ADE) }\end{array}$ & SI vs. NO & $t=-1,935$ & .054 \\
\hline
\end{tabular}

Es importante destacar algunos aspectos de los resultados derivados del análisis de las medias de los grupos comparados:

a) La mayoría de los grupos comparados sin diferencia significativa en las medias, tiene relación con las variables generales (Género, edad, tiempo de experiencia laboral, nacionalidad propia, de la madre y del padre, así como idioma materno (castellano vs. catalán)

b) Elestudio y/o dominio deidiomas aparece con diferencia significativa, especialmente entre quienes han estudiado mucho un idiomafrente a aquellos que lo han estudiado poco o nada, no encontrándose diferencia significativa entre los que han estudiado un idioma poco y los que no han estudiado nada.

c) Todaslas variables que tienen relación con la experiencia directa antelo internacional y/o multicultural aparecen con diferencia significativa, excepto la variable número de profesores extranjeros. 


\section{Comparación de la sensibilidad intercultural con la experiencia ante lo internacional y/o lo multicultural en perfiles extremos}

A fin de poder realizar un análisis estadístico comparativo de los perfiles extremos del continuo de experiencia con lo internacional y/o lo multicultural y ver si existe diferencia significativa o no frente al resultado de la escala de actitud (sensibilidad intercultural), se formaron dos grupos extremos de análisis. En un extremo estaría el perfil de alta experiencia y en el otro extremo el perfil de nula o escasa experiencia.

Para armar dichos perfiles extremos se consideraron siete variables de contexto de la encuesta, relacionadas con su vivencia personal, a saber:

- Vivir en el extranjero

- Participar en programas de intercambio al extranjero

- Viajar al extranjero

- Convivir con extranjeros

- Tener amigos extranjeros

- Estudiar idiomas extranjeros

- Participar en eventos relacionados con otras culturas

Se otorgó valor de un punto si han tenido la experiencia y 0 si no la han tenido, por lo que al sumar las puntuaciones obtenidas por cada uno de los alumnos en las diferentes variables, se obtiene un continuo de perfiles de experiencia que va del perfil 0 al perfil 7.

En un extremo del continuo estaría el perfil de alta experiencia multicultural (perfil 7 o que han puntuado en todas las variables) y en el extremo opuesto, el perfil 0 o de nula experiencia (que no han puntuado en ninguna de las variables). En la tabla 3 se detallan los dos perfiles que delimitan los extremos del continuo.

Tabla 3

Perfiles extremos

\begin{tabular}{|ll|}
\hline PERFIL DE ALTA EXPERIENCIA (4 al 7) & PERFIL DE NULA O ESCASA EXPERIENCIA (0 al 3) \\
\hline Haber vivido en el extranjero > 6 meses & NO haber vivido en el extranjero \\
\hline Haber participado en intercambio al extranjero & NO haber participado en intercambio al extranjero \\
\hline Haber viajado al extranjero > de 6 veces & NO haber viajado al extranjero \\
\hline Haber convivido con extranjeros & NO haber convivido con extranjeros \\
\hline Tener amigos extranjeros & NO tener amigos extranjeros \\
\hline $\begin{array}{l}\text { Haber estudiado al menos 2 idiomas extranjeros } \\
\text { (mucho) }\end{array}$ & $\begin{array}{l}\text { NO haber estudiado ningún idioma extranjero o } \\
\text { solo uno (poco) }\end{array}$ \\
\hline $\begin{array}{l}\text { Haber participado en al menos 2 tipos de eventos } \\
\text { (con cierta frecuencia) }\end{array}$ & \begin{tabular}{l} 
NO haber participado en ningún tipo de evento \\
\hline
\end{tabular} \\
\hline
\end{tabular}


Se formaron dos grupos extremos de análisis, en función de si obtenían puntos en cuatro o más de las variables que definen el perfil de experiencia multicultural (grupo 1) y aquellos que obtenían puntos en tres o menos de las variables (grupo 2).

De acuerdo con el análisis estadístico, SÍ existe diferencia significativa entrelos dos perfiles de grupos analizados, de lo que se puede deducir que existe relación entre la sensibilidad intercultural y la experiencia ante lo internacional y/o lo multicultural. De esta manera, podemos decir que el grupo de alumnos con una media de alta experiencia con lo internacional y/o lo multicultural, también tiene una media alta de sensibilidad intercultural. Ver síntesis de resultados en tabla 4.

\section{Tabla 4}

Comparación de medias entre grupos extremos (perfil 1 vs. perfil 2)

\begin{tabular}{|c|c|c|}
\hline $\begin{array}{c}\text { Media grupo perfil 1 (=>4) } \\
\text { Valor estadístico (escala Likert) }\end{array}$ & $\begin{array}{c}\text { Media grupo perfil 2 }(<4) \\
\text { Valor estadístico (escala Likert) }\end{array}$ & $\mathrm{P}=$ (significación al 0,05\%) \\
\hline $100.13(4.17)$ & $93.87(3.91)$ & .000 \\
\hline
\end{tabular}

Cabe resaltar algunos aspectos relacionados con la distribución de los integrantes de la muestra, según se detalla gráficamente en la figura 1.

Si se divide la muestra en tres grupos, el grupo inferior (perfiles 0 y 1) lo ocupa un $52 \%$ del alumnado encuestado; los perfiles 2 y 3 un $26 \%$ de la muestra y el perfil alto que ocupa la cúspide de la pirámide (los perfiles del 4 al 7), el 22\% restante.

La puntuación máxima del perfil son 7 puntos, los cuales fueron obtenidos por 4 alumnos de género masculino (todos parte del alumnado de nacionalidad propia extranjera y estudiantes del Programa de Intercambio ERASMUS).

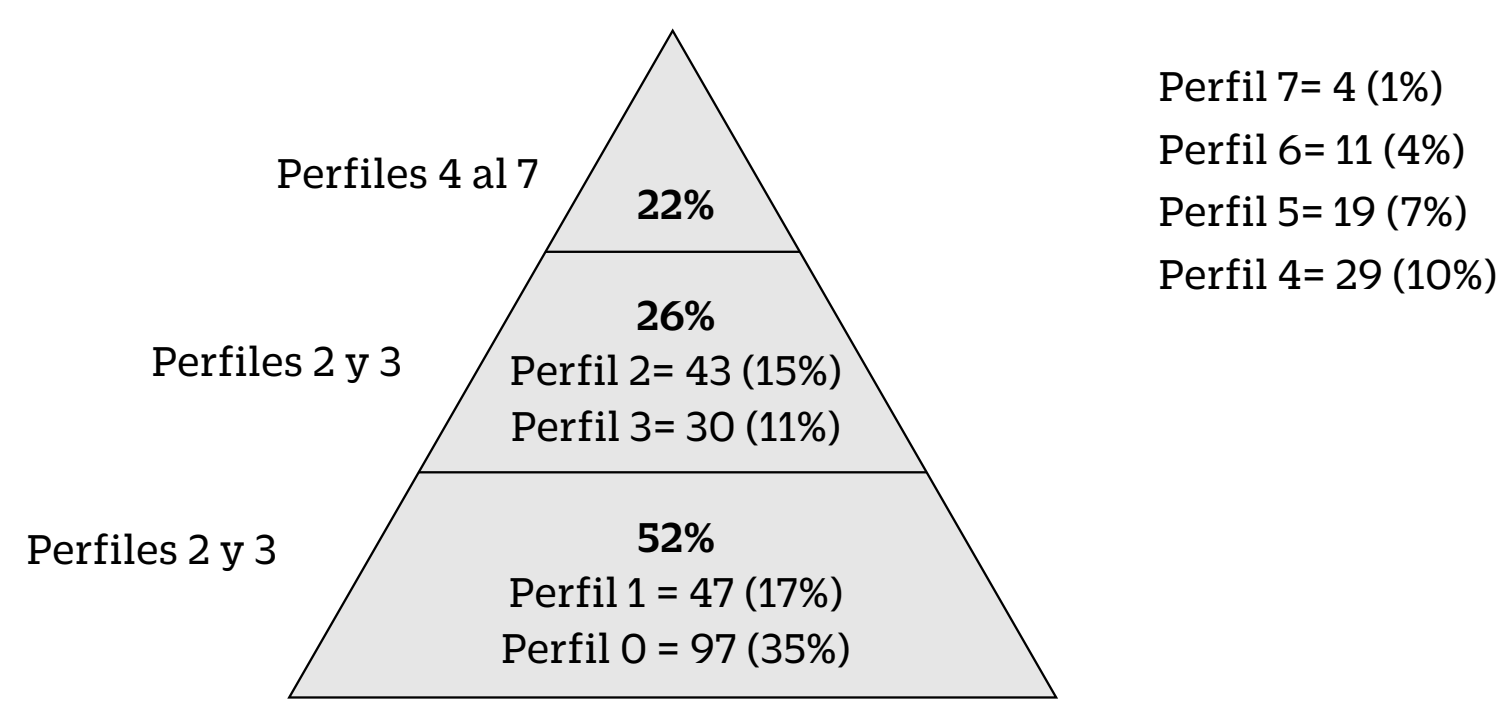

Figura 1. Distribución de alumnado encuestado por perfiles 
Al encontrar que los integrantes del perfil máximo son todos de nacionalidad propia extranjera y además estudiantes del Programa ERASMUS, se realizó un análisis descriptivo del alumnado extranjero que estaba en la muestra y se encontraron los siguientes aspectos que vale la pena destacar:

- $\quad$ No obstante que el alumnado extranjero es solo el 7\% de la muestra (20 personas), el 85\% de ellos (17) se ubican en el perfil alto (perfiles 4 al 7).

- De estos 17, 13 (76\%) pertenecen al Programa de Intercambio ERASMUS y el resto (4) no participan del programa ERASMUS, pero todos ellos tienen en común que han vivido más de tres años en el extranjero.

- En relación a las siete variables consideradas en el perfil de alta experiencia ante lo internacional y/o multicultural, el alumnado con nacionalidad propia extranjera, tiene la siguiente distribución, que bien vale la pena destacar:

- $\quad$ 94\% han viajado al extranjero más de seis veces

- $\quad 82 \%$ han convivido con extranjeros en la misma casa o piso

- $\quad 76 \%$ han participado en algún programa de intercambio al extranjero

- $\quad$ 76\% han estudiado mucho dos idiomas extranjeros, diferentes al idioma materno

- $\quad$ 70\% tienen amigos extranjeros entre sus mejores amigos

- $\quad$ 47\% han participado en al menos dos tipos de eventos relacionados con otros países o culturas (con cierta frecuencia)

- $\quad$ 41\% han vivido en el extranjero entre 7-12 meses y 29\% más de tres años

\section{DISCUSIÓN}

A la luz de los resultados del análisis de las características técnicas de la escala, puede concluirse que el instrumento traducido y adaptado es un instrumento con fiabilidad alta. También, de acuerdo con los resultados de los estudios validez de contenido de la escala, puede concluirse que el instrumento cuenta con validez de contenido. No obstante, se sugiere eliminar el ítem 15, ya que al eliminarlo el alpha aumentaría a un .8567, prácticamente la misma que tenía la escala original, o bien, hacer el intento con una nueva redacción de dicho ítem y ver el resultado que se obtiene en una nueva aplicación. Asimismo, se sugiere incluir dentro de los eventos relacionados con otras culturas, los eventos deportivos. Considero que con estos ajustes, el instrumento es válido y confiable para ser usado en el contexto universitario español.

Soy consciente de que la muestra se ha limitado a estudiantes de una sola universidad española, lo que supone una limitación, dado el alcance del fenómeno. Aun así, la muestra es suficientemente representativa de la población total y considero que con la validación del instrumento efectuada, estaría en condiciones para probarlo en otros contextos universitarios 
hispanoparlantes, tanto de universidades públicas como privadas, a fin de verificar su validez fuera del contexto español.

En cuanto al resultado de la medición de la variable sensibilidad intercultural (actitud del alumnado universitario hacia el hecho de interactuar con personas de diferente cultura), puede concluirse que el alumnado universitario encuestado tiene una actitud favorable en promedio hacia el hecho de interactuar con personas de diferente cultura, ya que el resultado se ubica en el nivel 4 "de acuerdo", con una mediana cercanía también al "de acuerdo", lo cual indica una actitud favorable en promedio.

De acuerdo al análisis efectuado entre la variable objeto de estudio (sensibilidad intercultural) vs. las variables de contexto del alumnado, creo que lo más relevante es constatar que todas las variables que tienen relación con la experiencia directa ante lo internacional y/o multicultural aparecen con diferencia significativa, excepto la variable número de profesores extranjeros.

Considero que el hallazgo más significativo del estudio surgió cuando se realizó el análisis de los perfiles extremos de experiencia, ya que al considerar solo los promedios había información que no quedaba visible, pero que al analizar las diferencias significativas de los grupos con perfiles de alta o nula o escasa experiencia ante lo internacional y multicultural apareció claramente la relevancia del componente vivencial en el desarrollo de la competencia. Al encontrar que SÍ existe diferencia significativa entre los dos perfiles de grupos analizados, se puede concluir que existe relación entre la sensibilidad intercultural y la experiencia ante lo internacional y/o lo multicultural. De esta manera, podemos decir que el grupo de alumnos con una media de alta experiencia con lo internacional y/o lo multicultural, es esperado que tenga también tiene una media alta de sensibilidad intercultural.

Estos resultados vienen a corroborar lo que varios autores han mencionado en sus estudios sobre el hecho de que el aprendizaje de la competencia intercultural es un proceso activo de aprendizaje permanente a lo largo de toda la vida (Taylor 1994), con un alto componente vivencial, es decir, que se lleva a cabo sobre todo a través de la vivencia o experiencia directa con "el otro diferente", sea en interacciones de uno a uno, o bien, grupales; sea en el propio país o en el extranjero (Anderson, 2000; Arthur, 2001).

Por último, no quisiera terminar sin hacer algunas reflexiones, creo pertinentes, con base en los resultados del perfil de experiencia del alumnado universitario encuestado.

El hecho de que el 52\% del alumnado universitario encuestado tenga un perfil de escasa o nula experiencia en ámbitos internacionales y/o multiculturales, plantea la cuestión de si ¿está el alumnado universitario suficientemente preparado para laborar en entornos empresariales multiculturales y/o internacionales?

El hecho de que el alumnado que se ubica en los perfiles bajos de la escala sea alumnado de nacionalidad española me lleva a preguntar si ¿es conveniente para el alumnado universitario 
actual, como potencial profesional de la Unión Europea y un mundo cada vez más global ubicarse en la parte inferior de la pirámide?

Los perfiles altos de la escala los ocupa el alumnado extranjero (85\%), no por el hecho de ser extranjeros, sino por contar con una experiencia acumulada que podría sintetizarse en a) una alta movilidad (viajes, programas de intercambio, vivir en el extranjero); b) convivencia o amistad con extranjeros, c) estudio de al menos dos idiomas extranjeros, así como d) participación frecuente en eventos relacionados con otras culturas o países.

El gran reto está en cómo motivar e incentivar al alumnado universitario español o de otras universidades hispanoamericanas para que paulatinamente adquiera mayor movilidad, estudie idiomas, participe en eventos relacionados con otras culturas y tenga mayores oportunidades de convivencia, lo cual le lleve a tener un perfil de alta exposición a lo internacional y/o multicultural, y que de esta manera se encuentre más sensible y preparado para diagnosticar, relacionarse y afrontar entornos laborales multiculturales.

Ante este reto, las instituciones de educación superior necesitan desarrollar contextos o entornos que permitan al alumnado universitario tener acceso a diversas estrategias de experiencia con lo internacional o multicultural, como son: a) vivir en el extranjero; b) viajar al extranjero; c) estudiar idiomas; d) participar en eventos de otros países o culturas y e) convivir con extranjeros, por lo que creo que dichas estrategias debieran tener una importancia prioritaria en cualquier programa de intervención educativa que pretenda el desarrollo de la competencia intercultural del alumnado universitario.

\section{REFERENCIAS}

Anderson, S. K., MacPhee, D. y Govan, D. (2000). Infusion of multicultural issues in curricula: A student perspective. Innovative Higher Education. 25(1), 37-57

Arthur, N. (2001). The multicultural counseling competencies of Canadian counsellors. Canadian Journal of Counseling. 35 (1), 36-48.

Bartolomé, M. (2002). Identidad y Ciudadanía. Un reto a la educación intercultural. Madrid. Narcea Ediciones Bertely Busquets, M. (enero 2011). Educación superior intercultural en México. Revista Perfiles Educativos. Vol.33 spe México Cano Barrios, J.; Ricardo Barreto, Carmen.; Del Pozo Serrano, Francisco. (2016) Competencia intercultural de estudiantado de educación superior: Un estudio en la Universidad del Norte (Barranquilla. Colombia). Revista Encuentros, Universidad Autónoma del Caribe, 14(2), 159-174. DOI: https://doi.org/10.15665/re.v14i2.734

Chen, G. y Starosta, W. J. (2000). The Development and validation of the Intercultural Sensitivity Scale. Reunión anual de la National Communication Association. (Seatlle, WA. Noviembre 8-12) (paper)

De Santos, F. J. (2004). Desarrollo de la competencia intercultural en alumnado universitario: una propuesta formativa para la gestión en empresas multiculturales (Tesis doctoral). Universidad de Barcelona. Barcelona, España.

Edwards Schachter, M. y López Santiago, M. (2008). Competencias comunicativas e interculturales y reforma curricular 
en el marco de la convergencia europea. Universidad Politécnica de Valencia. Revista Complutense de Educación, 19(2), 369-383.

Espinosa Romero, M. (2009). Los procesos de internacionalización empresarial y la gestión de personas. Fundación EOI (Escuela de Organización Industrial).

Ladrón de Guevara, S. (2014). La interculturalidad en el ámbito de la educación superior. Aula Intercultural. El portal de la educación intercultural. Recuperado de: https://goo.gl/a8vkVv

Martínez-Lirola, M. (enero-abril 2018). La importancia de introducir la competencia intercultural en la educación superior: Propuesta de actividades prácticas. Universidad de Alicante. Revista Electrónica Educare (Educare Electronic Journal), 22(1), 1-19

Mato, D. (2008). Coord. Diversidad Cultural e Interculturalidad en Educación Superior. Tendencias y Experiencias en América Latina. Caracas. Unesco-IESALC.

Mato, D. (2009). Coord. Instituciones interculturales de Educación Superior en América latina. Procesos de construcción, logros, innovaciones y desafíos. Caracas. Unesco-IESALC.

Mato, D. (2009a). Coord. Educación Superior, Colaboración Intercultural y Desarrollo Sostenible / Buen vivir. Experiencias en América latina. Caracas. Unesco-IESALC.

Mato, D. (2010). Diversidad Cultural e Interculturalidad en Educación Superior. Tendencias y Experiencias en América Latina. Unesco-IESALC. Boletín IESALC Informa. No. 212. Diciembre 2010.

Medina R. A.; Rodríguez M. A. e Ibáñez, A. coords. (2005). Interculturalidad, formación del profesorado y educación. España. Pearson Educación.

Mendoza, Blanca (2013). Las competencias interculturales. Aproximaciones críticas y analíticas a la construcción de sus significados dentro de la universidad veracruzana intercultural, sede Totonacapan (Tesis de maestría). Universidad Veracruzana Intercultural. México

Pascual-Ortega, D. A. (2013). Estrategias de interculturalidad en educación superior en México (Tesis de maestría). ITESO. Tlaquepaque, Jalisco.

Pozo-Vicente, Cristina; Aguaded-Gómez, José Ignacio (2012). El programa de movilidad ERASMUS: motor de la adquisición de competencias interculturales. Revista de Investigación Educativa, 30 (2), 441-458.

Taylor, E. W. (1994). Intercultural competency: A transformative learning process. Adult Education Quaterly, 44(3), 154-174

Villar, S. A. (2008). Vivir y trabajar en España: la competencia intercultural en el mundo de la empresa. Santander, España. Universidad de Cantabria.

RIDU / Revista Digital de Investigación en Docencia Universitaria / ISNN 2223-2516

(c) Los autores. Este artículo es publicado por la Revista Digital de Investigación en Docencia Universitaria del Área de Institutional Research and Effectiveness de la Dirección de Aseguramiento de la Calidad, Universidad Peruana de Ciencias Aplicadas. Este es un artículo de acceso abierto, distribuido bajo los términos de la LicenciaCreativeCommons Atribución-CompartirIgual 4.0 Internacional. ( http://creativecommons.org/licenses/by-sa/4.0/), que permite el uso no comercial, distribución y reproducción en cualquier medio, siempre que la obra original sea debidamente citada. 